\title{
Research Progress on Risk Factors and Prevention Strategies of Falls in Elderly Inpatients
}

\author{
Miaoqing Zhuang1, Jiamei Lì,*, Hongfang Chen², Yonghong Yang², Xia Song², Pei Li², Liang Shi², \\ Huanze Li ${ }^{1}$
}

${ }^{1}$ Shaanxi University of Chinese Medicine, Xianyang, Shaanxi, China.

${ }^{2}$ Shaanxi Traditional Chinese Medicine Hospital, Xi'an, Shaanxi, China.

\begin{abstract}
How to cite this paper: Miaoqing Zhuang, Jiamei Li, Hongfang Chen, Yonghong Yang, Xia Song, Pei Li, Liang Shi, Huanze Li. (2021) Research Progress on Risk Factors and Prevention Strategies of Falls in Elderly Inpatients. International Journal of Clinical and Experimental Medicine Research, 5(2), 101-105.

DOI: $10.26855 / \mathrm{ijcemr} .2021 .04 .002$
\end{abstract}

Received: January 10, 2021

Accepted: February 4, 2021

Published: February 25, 2021

*Corresponding author: Jiamei Li, female, nurse-in-charge, nursing management, Shaanxi Traditional Chinese Medicine Hospital, Xi'an, Shaanxi, China.

Email: $389973878 @ q q . c o m$

\begin{abstract}
The importance of falling is becoming increasingly prominent in the health problems of the elderly. Elderly inpatients are at high risk of falling, and falling is one of the important risk factors affecting the physical health and quality of life of the elderly. Based on the occurrence of fall will cause elderly patients with fear of falling, action impaired ability, function dependence, the economic burden and the negative impact of caregiver burden and so on, in the full understanding of the risk factors of elderly hospitalized patients, based on fall prevention strategies at home and abroad are reviewed, to provide personalized for senile inpatients, systematic fall prevention measures, and strengthen the safety consciousness of nursing staff, thus reducing the incidence of an elderly hospitalized stumble, protect the safety of senile inpatients, has become an important part of patient safety, as one of the important indexes of clinical nursing quality evaluation.
\end{abstract}

\section{Keywords}

Elderly Inpatients, Fall, Risk Factor, Prevent

According to the World Health Organization, a fall is when a person accidentally falls to the ground or floor or another low place. The World Health Organization defines a fall as an unintentional event that causes a person to fall to the ground or other lower plane. The fall problem is becoming more and more prominent in the elderly population, which is an important factor affecting the health of the elderly population. Research shows that in the global community, $30 \%$ of the elderly over 65 years old fall at least once a year, and the elderly fall probability increases with age, and the incidence of falls for the elderly over 80 years old is as high as $50 \%$ [1]. Falls are the fourth most common adverse event, not only causing physical and psychological harm to patients, such as prolonged hospital stay and increased medical costs; for hospitals, it can reduce patient satisfaction, increase medical disputes, and even affect the hospital's reputation.

Studies by foreign scholars have shown that there are different risk factors for hospitalized and community aged people. Falls in older patients is not an accident, but certain risk factors can be prevented and controlled. Therefore, it is particularly important to prevent falls in elderly inpatients. The purpose of this paper is to understand the risk factors of elderly inpatients, and to review domestic and foreign strategies for the prevention of falls, to provide a reference for future studies, and to improve the safety management of elderly inpatients.

\section{Risk factors for falls in elderly inpatients}

It is generally believed in academic circles that falling is a social epidemic caused by a combination of physiological, psychological, environmental, disease, and other comprehensive factors [2]. A large body of 
literature on fall risk and epidemiology suggests that falls in elderly inpatients are caused by a combination of risk factors. Not only the risk brought by their diseases but also the risk of falling caused by external factors should not be ignored. Early identification of risk factors for falls in elderly inpatients can help patients improve their awareness of risk factors identified during hospitalization and effectively avoid the occurrence of falls.

\subsection{Age and gender factors}

Age is an independent risk factor for falls, and the incidence of falls increases with age. With the increase of age, the physiological functions of the elderly decline, balance, and coordination ability also have different degrees of decline. Studies have shown that balance ability can be a predictor of falls [3, 4]. Vision plays an important role in postural balance in elderly patients, and studies have shown that falls in elderly patients aged 65 are significantly correlated with the decline of visual resolution [5]. A study of 2015 China health and retirement survey (CHARLS) data, screening of eligible 4,736 old people aged 60 and above as the research object is analyzed, the results show that the risk of falls in women more [6], which may be related to the low level of older women postmenopausal estrogen secretion, therefore, are more likely to develop osteoporosis [7], so the higher risk of falls.

\subsection{Time and place factors}

A retrospective analysis was conducted on the fall time of 12 inpatients in a third-grade first-class hospital [8]. The results showed that 6 out of 12 hospitalized patients fell in the toilet, with an incidence of $50 \%$. 7 patients experienced falls between 0.00 and 4.30, which may be associated with insufficient night shift nursing staff, high frequency of toilet visits, poor mental state, and the rest time of the family members. Also, the elderly night because of the position change and cause insufficient brain blood supply, standing instability, and lead to falling. This is similar to the research results of Zhao Guijuan [9].

\subsection{Disease factors}

The more chronic diseases older people had, the greater their risk of falling, the study reported. Stroke, hypertension, and diabetes are the main influencing factors of falls, and cerebellar insufficiency, arthritis, postural hypotension, anemia, Parkinson's disease are all related to falls [10].

\subsection{Drug factors}

Drug metabolism in elderly patients is slow and accumulation is easy to occur. Also, the elderly are more likely to have adverse reactions due to their sensitivity and tolerance to drugs than the young and middle-aged. When elderly patients take sedatives before surgery, they may experience cognitive decline, visual impairment, delirium, and, in severe cases, confusion, thus increasing the risk of falls in elderly patients [11]. Also, studies have shown that hypoglycemia in elderly patients after taking hypoglycemic drugs is likely to lead to falls, postural hypotension in elderly patients after taking hypertension drugs and heart-strengthening drugs is likely to lead to falls, and hyperkalemia and hypokalemia in some patients due to electrolyte disturbances caused by taking diuretics are likely to lead to falls [9].

\subsection{Environmental factors}

At present, most medical institutions focus on reducing environmental risk factors for falls. Environmental factors can not only independently increase the incidence of falls in elderly inpatients, but also contribute to the occurrence of falls together with other factors. The light in the ward is dim, the sight of elderly patients is not clear, the floor of the corridor is slippery, the carpet is loose, there are obstacles, the stairs are too narrow or the steps are too high, and the bathroom is not equipped with handrails, which can easily lead to falls.

\section{Interventions to prevent falls in the elderly}

\subsection{Intervention measures to prevent falls of the elderly abroad}

\subsubsection{Portable nursing stations outside wards can reduce the incidence of falls}

One intervention, called SITB, is to set up portable care stations in certain ward areas (consisting of four to six beds) to allow nurses to do their daily work while spending more time with patients. The portable care station consists of a desk with a pulley and a computer with a coded lock on a drawer for storing medical records. There are single rooms on each floor and a portable work station outside each ward. When the nurse is working on a portable workstation, keep the door of the ward open as much as possible for observation. Nurses are encouraged to 
work in SITB as much as possible, rather than doing routine work in a large nursing station [12].

\subsubsection{Video monitoring is helpful to reduce the incidence of falls}

After monitoring rehabilitated patients for 21 months, Cournan et al. [13] concluded that video monitoring can improve the safety of patients by reducing falls, reduce the use and corresponding costs of caregivers, and improve the satisfaction of patients, their families, and staff. The technician monitors the patient remotely and can talk to the patient or contact the staff.

\subsubsection{Fitness games can help improve the physical and cognitive functions of the elderly}

Fitness game interventions such as the Nintendo Wii and Xbox Kinect can improve physical and cognitive function in older adults. While various common exercises can improve strength and balance, poor adherence to the program can hinder their effectiveness. Choi et al. [14] found that to make the exercise program successful, the program should be enjoyable and easy to execute. Athletic interventions such as handball games and slalom skiing helped improve balance compared with no intervention at all.

\subsubsection{Use the six Sigma method to reduce the incidence of falls}

The six Sigma tool DMAIC (definition, measurement, analysis, improvement, and control) helped King Fahd hospital reduce the fall rate from 6.57 to 1.91 per 1,000 patient days in three months. The staff put forward and implemented eight strategies, including the understanding and communication of patients' risk of falling, staff training, unified understanding, and the use of bedside alarm [15].

\subsection{Domestic intervention measures to prevent falls of the elderly}

\subsubsection{Traditional exercise therapy}

Exercise is one of the more effective measures to prevent falls in old age. Research shows that as a single intervention strategy to prevent falls in old age, exercise intervention is the most effective method. Chinese scholars have studied whether taijiquan exercise can reduce falls in patients with Parkinson's syndrome. The Tai Chi group practiced Yang's Tai Chi for 60 minutes three times a week for 12 weeks. Patients in the Tai Chi group showed better balance compared to the control group. Following up for 6 months, the Tai Chi group was found to have a lower incidence of falls $(21.6 \%$ vs $48.7 \%)$. The study suggested that this method could be extended to non-Parkinson's disease patients [16].

\subsubsection{Balance training}

Studies have shown that balance training can reduce patients' fall effectiveness, improve patients' self-living ability, reduce elderly patients' fear of falling, and thus enhance their confidence in not falling [17]. This is similar to the results of Halvarsson et al. [18], that balance training improves the level of falling self-efficacy. This suggests that nursing staff should encourage elderly patients to carry out humidity balance training to enhance fall-related self-efficacy, to reduce the risk of falling.

\subsubsection{Psychological intervention}

Fear of falling (FOF), also known as the Fear of falling, refers to the imaginary decline in self-efficacy that occurs during an activity to avoid falling. FOF is prevalent in over 65 elderly patients [19]. Guo spray of molten steel such as [20] 60 lee stroke patients were randomly divided into a control group and experimental group, two groups adopt routine rehabilitation training and observation group based on the conventional training before the training, training and after training to give different psychological intervention, intervention, $40 \mathrm{~min} /$ time intervention 11 times, a total of 4 weeks intervention, the results show that the falling effectiveness and walking training score in the observation group were higher than the control group. FOF itself is a special form of anxiety. Elderly patients with negative emotions such as anxiety or depression should be provided with targeted psychological counseling to reduce their negative emotions and improve their cognition of falling.

\subsubsection{Dual-mode health education}

Dual-mode health education integrates health beliefs into nursing work and fully implements targeted health education according to the overall positive and intermittent nature of nursing procedures. Zhang Yi et al. [21] randomly divided 240 elderly hospitalized patients into the control group and the intervention group. The control group was given conventional health education on fall prevention, while the intervention group was given dual-mode health education. Health education is carried out according to the steps of evaluation-programme-Implementation- evaluation. The results showed that, after the intervention, the knowledge level, health belief level, and health behavior level of fall prevention in the observation group were all changed in 
the control group, which was of great significance for the prevention of falls in hospitalized patients.

\section{Suggestions}

To help medical institutions prevent falls and avoid injuries caused by falls, the following suggestions are made: to prevent falls requires the attention of leaders, requires a systematic, data-driven way to reduce the risk of falls, and continuously improves the specific environment and improves the quality of specific groups.

\subsection{Raise awareness to prevent fall injuries}

Communicate safety information to all levels of clinical and non-clinical personnel.

Incorporate safety precautions into long-term patient care and education through changes in management principles and tools, including how to create conditions, how to make methods easy, how to empower employees, how to ensure accountability, how to get leadership support and commitment, and how to continuously improve. To strongly support change in management, the project leader should be empowered to ensure adequate equipment and resources, including staffing and preventive devices (such as alarms), and a clinically experienced staff that can influence stakeholders while facilitating the provision of care to patients.

\subsection{Establish an interdisciplinary fall-prevention team and conduct assessment and training for team members}

Ensure that health facilities are well equipped to reduce the risk of fall injuries. It is everyone's responsibility to reduce fall injuries. Caregivers, physicians, cleaning staff, its staff, family members of patients, pharmacy staff, physical and occupational therapy staff, quality and risk management staff, and other relevant stakeholders are all responsible.

\subsection{Use standard, effective assessment tools to identify fall risk factors}

Such as the Morse fall assessment scale or Hendrich II fall risk assessment model, best can integrate evaluation tool to use in electronic medical records. In addition to tool use, there should be a comprehensive, personalized assessment of the risk of falls and injuries. The assessment should include the patient's age, sex, cognitive status, and functional level. Train health care workers on the use of tools to ensure credibility among evaluators.

\subsection{Based on the identification of the risk of falls and injuries, develop personalized care plans and implement interventions for specific patients, populations, and environments}

To some extent, all patients are at risk of falling, so specific care must be developed for each patient's specific type of risk to reduce the risk of falling. Real risk assessment is not just about screening, but also about guiding clinicians in developing prevention strategies for identified risk factors. For example, since 2008, the Veterans Health Administration program has been evaluating patients for falls, risk of injury, and a history of fractures.

\subsection{Adopt and standardize effective practices and interventions}

Standardized information transfer and communication processes are adopted among medical and care personnel to convey patients' risk of falling, including the identification of specific risk areas and measures to reduce the risk of falling for specific individuals. For example, according to different situations, the following measures can be adopted for information communication: write down the information related to falls on the whiteboard to remind the medical staff of each shift to pay attention; enter warnings, tasks, records, and prompts into electronic medical records; bedside rotations of patients with fall-related problems. Each patient is taught one to one by the bedside using educational materials, including risk and cause of falls, prevention strategies, goal setting, and assessment.

\section{Summary}

In the occurrence of falls in the prevention, nursing staff should strengthen the early identification, actively take effective means of prevention. Although the incidence of falls has decreased significantly after the implementation of nursing measures, the causes of falls in elderly inpatients are complex and changeable, and the physical and mental damage and social and economic pressure brought by falls to elderly inpatients are great. Therefore, how to carry out the management of elderly inpatients and prevent the occurrence of falls in elderly inpatients more effectively deserve further research. 


\section{Funding}

Project name: A hospital-level scientific research project of Shaanxi Academy of Traditional Chinese Medicine (Project No.: 2018-30).

\section{References}

[1] CAI lun, cen, Lin zhou Zi, et al. (2008). Advances in public health research on falls in the elderly [J]. Chinese Journal of Gerontology, 2008, 38(09): 2265-2268.

[2] Xiao Chunmei, Zhou Julin, Li Yang, et al. (2002). Foreign research progress on factors related to falls in the elderly [J]. Chinese Clinical Rehabilitation, 2002(07): 1014-1015.

[3] Shi Yu, Zhao Hong, Han Jinhua, et al. (2014). Investigation on knowledge, attitude, and behavior of partner caregivers of patients with cerebrovascular disease [J]. Nursing Research, 2014, 28(15): 1821-1823.

[4] Yu Aihong, Yuan Wanhuan, Lan Limei, et al. (2010). Safety hazard Analysis and Nursing Countermeasures for inpatient stroke patients [J]. Journal of Youjiang Medical College for Nationalities, 2010, 32(02): 280-281.

[5] Shi Jing, Yao Huiqing, Tao Yongkang, et al. (2016). Chinese journal of geriatrics, 2016, 35(05): 551-555. (in Chinese with English abstract)

[6] Lu Junying, Guo Dongxia, Li Fangwei, et al. (2020). Journal of Zhengzhou University (medical edition), 2020, 55(05): 662-667.

[7] Saari, P., Heikkinen, E., Sakari-Rantala, R., et al. (2007). Fall-related injuries among initially 75- and 80-year old people during a 10-year follow-up [J]. Arch Gerontol Geriatr, 2007, 45(2): 207-15.

[8] Liu Min. (2013). Analysis of causes of falls in elderly inpatients and Discussion of preventive measures [J]. Contemporary Nurses (the following ten-day issue), 2013(06): 100-101.

[9] Zhao Guijuan. (2014). Analysis of causes of falls and falls in 17 elderly inpatients and preventive measures [J]. China Rural Health, 2014(15): 48.

[10] Ji Yanhu, Li Liping, Chen Wentao. (2012). Chinese Journal of Disease Control, 2012, 16(01): 22-24. (in Chinese with English abstract)

[11] Lin Jianyu, Zhang Ying, Sun Chao, et al. (2017). Causes analysis and countermeasures of falls in elderly patients induced by sedative and hypnotic drugs during hospitalization [J]. Chinese Journal of Geriatrics, 2017, 36(06): 627-631.

[12] Ali, U. M., Judge, A., Foster, C., et al. (2018). Do portable nursing stations within bays of hospital wards reduce the rate of inpatient falls? An interrupted time-series analysis [J]. Age Ageing, 2018, 47(6): 818-824.

[13] Cournan, M., Fusco-Gessick, B., Wright, L. (2018). Improving Patient Safety Through Video Monitoring [J]. Rehabil Nurs, 2018, 43(2): 111-115.

[14] Choi, S. D., Guo, L., Kang, D., et al. (2017). Exergame technology and interactive interventions for elderly fall prevention: A systematic literature review [J]. Appl Ergon, 2017, 65: 570-581.

[15] Kuwaiti, A. A., Subbarayalu, A. V. (2017). Reducing patients' falls rate in an Academic Medical Center (AMC) using Six Sigma "DMAIC" approach [J]. Int J Health Care Qual Assur, 2017, 30(4): 373-384.

[16] Ji Suqiong, Mao Zhijuan, Yang Qingmei, et al. (2016). Effect of taijiquan exercise on Parkinson's disease [J]. China rehabilitation, 2016, 31(01): 51-53.

[17] Li Ying, Cheng Yun, Zhao Lilong, et al. (2014). Effect of Otago exercise on fear of falling in elderly stroke patients [J]. Chinese journal of nursing, 2014, 49(12): 1442-1447.

[18] Halvarsson, A., Franzen, E., Stahle, A. (2015). Balance Training with multi-task Exercises improves fall-related self-efficacy, Gait,balance performance, and physical function in older adults with osteoporosis: a randomized controlled trial [J]. Clin Rehabil, 2015, 29(4): 365-75.

[19] Schmid, A. A., Van Puymbroeck, M., Knies, K., et al. (2011). Fear of falling among people who have sustained a stroke: a 6-month longitudinal pilot study [J]. Am J Occup Ther, 2011, 65(2): 125-32.

[20] Guo Ganghua, Yan Yingying, Li Zhe. (2014). (in Chinese) Effects of psychological intervention on balance function and walking ability of stroke patients with fall [J]. Chinese Journal of Rehabilitation Medicine, 2014, 29(04): 357-359.

[21] Zhang Yi, Chen Weiyong. (2019). Effect of dual-mode health education on the prevention of falls in hospitalized elderly patients [J]. Practical preventive medicine, 2019, 26(04): 477-480. 